\title{
Magnetic Anisotropy - How much is enough for a permanent magnet?
}

R. Skomski ${ }^{1}$ and J. M. D. Coey ${ }^{2,3}$

${ }^{1}$ Department of Physics and Astronomy and Nebraska Center for Materials and Nanostructures, University of Nebraska, Lincon NE 68588, USA.

${ }^{2}$ School of Physics and CRANN, Trinity College, Dublin 2, Ireland.

${ }^{3}$ School of Materials Science and Engineering, Beihang University, Beijing 100191, China.

\begin{abstract}
Materials choices for permanent magnets are analyzed in terms of energy product, anisotropy and hardness parameter. Energy product is the main consideration for permanent magnets, because the purpose of a magnet is to store magnetostatic energy and create as much flux as possible in the surrounding space. Magnet processing is easiest if the hardness parameter is significantly greater than one. The anisotropy requirement becomes increasingly stringent for large $M_{\mathrm{s}}$ and $K_{1}$ values of 1 to $2 \mathrm{MJ} / \mathrm{m}^{3}$. Some potential new magnets and alternative strategies to develop magnetic hardness are discussed.
\end{abstract}




\section{Introduction}

Permanent magnets are of growing importance in many areas of technology [Error! Reference source not found.-4,]. Their unique feature, quantified by the energy product $(B H)$, is their ability to store magnetostatic energy. Cumbersome, low-energyproduct bar and horseshoe magnets with energy products of about $1 \mathrm{~kJ} / \mathrm{m}^{3}$ were the best that could be achieved 100 years ago. Shapes were dictated by the low coercivity $H_{\mathrm{c}}$ of the ferromagnetic materials available at that time, which limited the tolerable demagnetizing field in the second quadrant of the $M(H)$ hysteresis loop where the working point of a magnet is inevitably located. A commonly-used approximation is to assume the demagnetizing field $H_{\mathrm{d}}$ is uniform and opposite in direction to the magnetization,

$$
\boldsymbol{H}_{d}=-\mathcal{D} \boldsymbol{M}
$$

where the demagnetizing factor $\mathcal{D}$ is a number between 0 and 1 that depends on the magnet shape. Hard steels, with a magnetization of $1.5 \mathrm{MAm}^{-1}$, and coercivity $H_{\mathrm{c}}$ of no more than $30 \mathrm{kAm}^{-1}$ could only retain their magnetization if $\mathcal{D}<0.02$.

Magnetic anisotropy is given, to first order, by the expression

$$
E_{\mathrm{a}}=K_{1} \sin ^{2} \theta
$$

where $\theta$ is the deviation of $\boldsymbol{M}$ from its easy direction when $K_{1}$ is positive. It is often represented by an 'anisotropy field' $H_{\mathrm{a}}=2 K_{1} / \mu_{0} M$, although this approximation is only strictly valid for an anisotropic, single-domain ferromagnet when $\theta$ is small. The sum of the demagnetizing factors for the three principal directions of a body is 1 , so when the magnet has an axis of symmetry $\mathcal{D}_{\perp}=1 / 2(1-\mathcal{D})$. It follows that the magnetostatic energy density $E_{\mathrm{ms}}=1 / 2 \mu_{0} \boldsymbol{M} \cdot \boldsymbol{H}_{\mathrm{d}}$ can be written in the form $1 / 4 \mu_{0} M^{2}(1-3 \mathcal{D}) \sin ^{2} \theta+$ const. The anisotropy constant in (2) that corresponds to the magnet's shape is

$$
K_{1}^{\mathrm{s}}=1 / 4 \mu_{0} M^{2}(1-3 \mathcal{D})
$$


Shape anisotropy is only fully effective in regions where the magnetization remains uniform and rotates coherently without breaking up into domains.

In a sequence of advances beginning in the 1930s, shape anisotropy was internalized in a series of two-phase alloys composed of nanoscale acicular inclusions of ferromagnetic Fe-Co in a matrix of Ni-Al. These alloys are cast and thermally-treated in a magnetic field to create the necessary oriented nanostructure by spinodal decomposition. A range of alnicos of different composition with alloy additions such as $\mathrm{Cu}, \mathrm{Nb}$ or $\mathrm{Ti}$ have magnetization in the range $0.6-1.2 \mathrm{MAm}^{-1}$ and coercivity of $50-160 \mathrm{kAm}^{1}$. Maximum energy products of magnets made from these materials range from 40 to 80 $\mathrm{kJm}^{-3}$. Alnicos remained the champion permanent magnets until the advent of $\mathrm{SmCo5} 50$ years ago, and they are still produced in significant quantities for niche applications where the high Curie temperature and excellent thermal stability of the Fe-Co is valued. However, the permanence of their magnetism is conditional on shape. The coercivity of oriented Alnico magnets is insufficient for them to retain their magnetization in shapes where $\mathcal{D}$ is greater than about 0.3 , so they are usually fabricated in the form of short bars or squat horseshoes. The shape anisotropy Eq (3) on which they rely could at most deliver an anisotropy field of only half the magnetization. The anisotropy field $H_{\mathrm{a}}$ sets an upper limit on the possible value of $H_{\mathrm{c}}$, and the coercivity in alnicos is always much less that $H_{\mathrm{a}}$, because the reversal is incoherent [2].

The shape barrier was broken in the mid 20th century thanks to anisotropy from a different source - magnetocrystalline anisotropy. There is a small dipolar 'two-ion' contribution of magnetostatic origin, but the main source is the 'single ion', electrostatic crystal field interaction acting in conjunction with spin-orbit coupling. Now there is a requirement that the crystal structure itself be uniaxial. The first magnets that retained their magnetization regardless of shape were made of the $L 1_{0}$ compound $\operatorname{CoPt}[2,5,6]$ and the hexagonal ferrite $\mathrm{BaFe}_{12} \mathrm{O}_{19}$ [7]. Later it was realized that the rare-earths were excellent sources of magnetocrystalline anisotropy and all today's high-performance permanent magnets are made from rare-earth transition-metal (RE-TM) intermetallics such as $\mathrm{Nd}_{2} \mathrm{Fe}_{14} \mathrm{~B}$ [8-10], $\mathrm{Sm}_{2} \mathrm{Co}_{17}$ [11], and $\mathrm{Sm}_{2} \mathrm{Fe}_{17} \mathrm{~N}_{3}$ [12]. These materials have led to an increase of energy product by another order of magnitude, to about $460 \mathrm{~kJ} / \mathrm{m}^{3}$, thereby 
enabling many new applications in motors, actuators, generators and static field devices. that have driven the annual permanent magnet market to a level of almost 10B $\$$, well over half of which is accounted for by 100,000 tonnes of rare earth permanent magnets. The balance of the total annual production of $1 \mathrm{M}$ tonnes is almost entirely made up of the hexagonal ferrites $\mathrm{BaFe}_{12} \mathrm{O}_{19}$ and $\mathrm{SrFe}_{12} \mathrm{O}_{19}$, which are cheap to produce, and offer energy products of up to $40 \mathrm{kJm}^{-3}$. There is a wide gap between the performance of ferrite or alnico on the one hand and Nd-Fe-B or Sm-Co on the other. There is an opportunity to fill the gap if a new magnet with an appropriate price/performance ratio of not more than about $1 \$ / \mathrm{J}$ can be found $[13,14]$.

Concerns about rare-earth supplies following the crisis in 2011 have sparked interest in new materials made entirely or mostly from less critical and cheaper elements, such as Fe, Co, Mn, Al, Bi, Zr, and W. Substantial funding in Japan, the US and Europe led to a revival of in permanent magnet research, which had fallen dormant after a flurry of activity in the 1980 s and 1990 s following the discovery of $\mathrm{Nd}_{2} \mathrm{Fe}_{14} \mathrm{~B}$. New materials are being considered, and old ones reconsidered for their permanent magnet potential. [15-20]. Since a reasonably large magnetization coupled with uniaxial anisotropy seems to be indispensable, it is appropriate to raise the question of just how much anisotropy is enough?

Figure 1 is a plot of anisotropy energy $K_{1}$ in $\mathrm{kJm}^{-3}$ versus saturation polarization $\mu_{0} M_{\mathrm{s}}$ in $\mathrm{T}$ for a wide range of magnetic materials with Curie temperatures well above room temperature, which exhibit uniaxial anisotropy of magnetocrystalline or shape origin. We will use the figure as a basis for our subsequent discussion. For the moment, we will simply introduce the magnetic hardness parameter

$$
\kappa=\sqrt{ }\left(K_{1} / \mu_{\mathrm{o}} M_{\mathrm{s}}^{2}\right)
$$

and mention that $\kappa>1$ is an empirical rule of thumb for a material to have a chance of resisting self-demagnetization when fabricated into any possible shape. The solid line on the plot is $\kappa=1$. 


\section{Concepts, Definitions and Limits}

\subsection{Energy Considerations}

The primary purpose of permanent magnets is to store magnetostatic energy. Magnets store only a little energy - a kilogram of Nd-Fe-B stores less energy than a milligram of chocolate - but they store it in a very useful form. To evaluate the magnetostatic energy contributions when no currents are present, it is convenient to start from the identity $1 / 2 \int \boldsymbol{B} \cdot \boldsymbol{H} \mathrm{d} V=0$ [2], where the integration extends over all space [21]. Splitting this integral into parts inside the magnet (index $I$ ) and outside the magnet where $\boldsymbol{B}=\mu_{\mathrm{o}} \boldsymbol{H}$ (index $A$ ) yields the sought-for external magnetostatic energy

$$
E_{\mathrm{A}}=1 / 2 \int_{\mathrm{A}} \mu_{\mathrm{o}} H^{2} \mathrm{~d} V=-1 / 2 \int_{\mathrm{I}} \boldsymbol{B} \cdot \boldsymbol{H} \mathrm{d} V
$$

This equation is normally interpreted as $(B H)=2 E_{\mathrm{a}} / V$ where the energy product $(B H)=$ $V^{-1} \int_{\mathrm{I}} \boldsymbol{B} \cdot \boldsymbol{H} \mathrm{d} V$ is twice the energy stored in the stray field of a magnet [22]. Since $\boldsymbol{B}=$ $\mu_{\mathrm{o}}(\boldsymbol{H}+\boldsymbol{M})$ and $\boldsymbol{H} \approx-\mathcal{D} \boldsymbol{M}$ in the absence of any external field (Eq 1), it follows that

$$
(B H)=\mu_{\mathrm{o}} \mathcal{D}(1-\mathcal{D}) M_{\mathrm{s}}^{2}
$$

where we have assumed that $M=M_{\mathrm{s}}$. This equation shows clearly that energy product is a shape-dependent property of a magnet not a materials parameter. It is instructive to discuss Eq. (6) for different shapes. Thin films with perpendicular and in-plane magnetization have $\mathcal{D} \approx 1$ and $\mathcal{D} \approx 0$, respectively, and both correspond to vanishing energy products. Figures 2(a-b) illustrate why: uniformly magnetized thin films produce no stray field outside the magnet, except at the edges, and most of the magnetic material is wasted. Similar considerations apply to horseshoe shapes such as the toroid in Fig. 1(c), characterized by $\mathcal{D} \approx g / L$, where $g$ is the gap width and $L$ is the contour lengths of the flux lines in the toroid.

Maximizing the energy product of Eq. (6) with respect to the shape of the magnet, $\partial(B H) / \partial \mathcal{D}=0$, yields $\mathcal{D}=1 / 2$. This corresponds to a compact magnet with height 
approximately equal to radius, as in Fig. 1(d). This shape yields $(B H)_{\mathrm{MAX}}=1 / 4 \mu_{0} M_{\mathrm{s}}^{2}$, the maximum possible energy product that can be achieved, from a perfectly rectangular $M(H)$ loop. The analysis of idealized rectangular $M(H)$ hysteresis loops to deduce the energy product from parallelogram-shaped $B(H)$ loops when $\mathcal{D}=0,1 / 2$ and 1 is illustrated in Fig. 3. The energy product is defined at the working point where the load line with permeance $B / H$ defined for the magnet in the absence of any applied field intersects the loop. Permeance depends only on magnet shape.

'Energy products' reported in the literature are frequently based on measurements of the hysteresis loops of uniformly magnetized thin films or powders. These are misleading. For thin films, there may be a nice hysteresis loop, but the energy product $(B H)$ defined at the working point is practically zero, because it is at $B=0$ for the $\mathcal{D}=1$ loop and at $H=0$ for the $\mathcal{D}=0$ loop. The nominal energy product deduced from such loops does not represent energy stored by the magnet, but energy derived from the external field source. There is only a significant stray field when a film is magnetized in domains that are comparable in width to the film thickness, as in magnetic recording media, or if thousands of films can be stacked to make a roughly equiaxed stack.. Note that, in principle, the minimum coercivity required to achieve the maximum value $(B H)_{\text {MAX }}$ for the ideal loop for a shape with $\mathcal{D}=1 / 2$ is $1 / 2 M_{\mathrm{s}}$ on the $B(H)$ loop, where coercivity is denoted as ${ }_{\mathrm{B}} H_{\mathrm{c}}$ to distinguish it from the coercivity $H_{\mathrm{c}}$ on the $M(H)$ loop.

The problem of powders, which are usually measured in open circuit in a vibratingsample magnetometer is different. The magnetization quoted is usually based on the mass of the powder, and converted to volume based on the powder density. The procedure yields an 'energy product' that would apply to a fully-dense magnet made from the powder, yet such a magnet and an assembly of separated particles will behave quite differently. The energy product for a practical magnet with $70-80 \%$ of the X-ray density may be only half that measured on the powder.

Although the stored magnetostatic energy per unit magnet volume, given by the energy product, is relevant for most applications, there may be some cases, particularly in aerospace, where the magnetostatic energy per unit magnet mass is more pertinent. A material with half the density of $\mathrm{Nd}-\mathrm{Fe}-\mathrm{B}$ or $\mathrm{SmCo}$, but more than half the energy product would be useful; regrettably, no such gap magnet yet exists. [14]. 


\subsection{Anisotropy and Coercivity}

An ideal magnet exhibits a rectangular hysteresis loop, the magnetization $M$ remains equal to $M_{\mathrm{s}}$ until coercive field $H_{\mathrm{c}}$ is reached in the second quadrant, when it switches to $M_{\mathrm{s}}$. In order to reach the theoretical maximum energy product with $\mathcal{D}=1 / 2, \quad(B H)_{\mathrm{MAX}}=$ $1 / 4 \mu_{\mathrm{o}} M_{\mathrm{s}}{ }^{2}, H_{\mathrm{c}}$ must be greater than $M_{\mathrm{s}} / 2$. In the absence of global shape anisotropy, the coercivity of a magnet can never exceed the anisotropy field, the value of coercivity corresponding to uniform magnetization reversal by coherent rotation; generally it is much less, at best about $25 \%$ of $H_{\mathrm{a}}$. This condition requires that $2 K_{1} / \mu_{0} M_{\mathrm{s}}>M_{\mathrm{s}} / 2$, or from Eq 4, $\kappa>1 / 2$. We see from Eq (3) that internal shape anisotropy can never exceed $1 / 4 \mu_{0} M_{\mathrm{s}}^{2}$ since $\mathcal{D}>0$. Hence, for internal shape anisotropy $\kappa<1 / 2$. It is therefore impossible to approach an ideal hysteresis loop with shape anisotropy alone. Magnetocrystalline anisotropy is indispensable.

Another limit is the anisotropy required for a magnet to resist demagnetization, whatever its shape. Now the anisotropy field must exceed the demagnetizing field, Eq (1). Since $\mathcal{D}<1$, the condition is $2 K_{1} / \mu_{0} M_{\mathrm{s}}>M_{\mathrm{s}}$ or $\kappa>1 / \sqrt{ } 2$.

It is always a formidable challenge to engineer coercivity in a new magnetic material, and the optimization process may take 20 years or more. The root of the problem is that the fully-magnetized state is metastable (except in a nanoparticle). A vortex or domain state is more stable because it reduces the energy in the stray field. Sophisticated microstructures have to be developed to impede the nucleation and growth of reverse domains, which usually involves separating oriented grains of the hard uniaxial ferromagnet from each other by a nonmagnetic grain-boundary phase. The goal to make the hard phase as dense and well-oriented as possible. Energy product scales as the square of the magnetization, hence as the square of the density of the hard phase in the magnet. The magnetization of an array of randomly-oriented uniaxial grains is only half that of a fully-oriented array, so the greatest achievable energy product is reduced by three quarters.

We are approaching the point where it is possible to simulate realistic microstructures [23], but it is still convenient to parameterize coercivity in terms of the empirical Kronmüller equation [24] 


$$
H_{\mathrm{c}}=\alpha 2 K_{1} / \mu_{\mathrm{o}} M_{\mathrm{s}}-\mathcal{D}_{\mathrm{eff}} M_{\mathrm{s}}
$$

where the dimensionless factor $\alpha$ multiplies the anisotropy field and $\mathcal{D}_{\text {eff }}$ is an effective local demagnetizing factor. $\alpha$ reflects the magnet's microstructure, including pinning and nucleation centers, and it is always much smaller than one. In well-processed magnets, it is usually in the range $0.1-0.3$. D $\mathcal{D}_{\text {eff }}$ accounts for the effect of local dipolar interactions on the coercivity, for example by creating stray fields near grain boundaries. It is unrelated to the macroscopic demagnetizing factor $\mathcal{D}$ and may have either sign. It is sometimes of the order of $1 / 3$, but in anisotropic sintered $\mathrm{Nd}_{2} \mathrm{Fe}_{14} \mathrm{~B}$ and $\operatorname{Pr}_{2} \mathrm{Fe}_{14} \mathrm{~B}$ magnets values may be close to or even larger than 1 , and in the case of alnico, $\mathcal{D}_{\text {eff }}$ is actually negative unless the shape anisotropy $K_{1}{ }^{\mathrm{s}}(\mathrm{Eq} 4)$ is included in the first term.

Magnetocrystalline anisotropy due to the crystal field interaction is largest for heavy elements such as rare earths, whose $4 f$ orbital moments are unquenched, and rigidly coupled to the spin $[2,8]$. The variation of rare-earth anisotropy energy across the $4 f$ series is well-understood, but there are no well-defined rules for the $3 d, 4 d$, and $5 d$ anisotropies as a function of the atomic number (or $d$-band filling $n$ ). The anisotropy tends to oscillate as a function of $n$, and depends on Fermi-level-dependent $k$-space summations [25].

The crystal field interaction depends on the uniaxial deformation of the site occupied by the magnetic atom, the electronic structure and the quadrupole moment of the atom itself. For $3 d$ atoms, the largest value, $6.5 \mathrm{MJm}^{-3}$ is found in YCo5. The values for magnetic rare earths can be several times greater.

\subsection{Magnetic hardness}

The dimensionless hardness parameter $\kappa=\left(K_{1} / \mu_{\mathrm{o}} M_{\mathrm{s}}{ }^{2}\right)^{1 / 2}$ captures the potential for a given material with adequate magnetization $M_{\mathrm{s}}$ to be developed into a compact permanent magnet. Figure 1 summarized the anisotropy and magnetization of a wide range of uniaxial materials, and Table 1 lists some numerical values of magnetization, anisotropy, and hardness for compounds of interest in permanent magnetism. Very large hardness 
parameters and coercivities can easily be achieved in materials with moderate anisotropy and little magnetization; $\alpha \mathrm{Fe}_{2} \mathrm{O}_{3}$ is a good example. It is a weak ferromagnet with a polarization $\mu_{0} M_{\mathrm{s}}$ of only $3 \mathrm{mT}$ and $K_{1}=9 \mathrm{kJm}^{-3}$, but $\kappa$ is eight times greater than it is for SmCos! Hematite is a hard magnetic material, but in no way is it a useful permanent magnet.

The parameter $\kappa$ can also be increased in magnets that are not fully aligned, permitting fabrication of magnet shapes with an unfavourable demagnetizing factor, at the expense of a greatly-reduced energy product. For randomly-aligned easy axes $\langle K\rangle=$ $1 / 2 K_{1}$ but $\left\langle M^{2}\right\rangle=1 / 4 M_{\mathrm{s}}^{2}$, so $\kappa$ is increased by $\sqrt{ } 2$. This approach has been used to make discshaped magnets from alnico 8 .

We classify ferromagnetic materials as hard, semi-hard and soft with dividing lines between the categories set, somewhat arbitrarily, at $\kappa=1$ and $\kappa=0.1$. Tetragonal $\mathrm{CrO}_{2}$, which is semi-hard with $\kappa=0.32$, owed its success as a magnetic recording medium to the possibility of fabricating it in acicular form, with a sufficient external shape anisotropy to maintain its magnetization. Likewise for $\gamma \mathrm{Fe}_{2} \mathrm{O}_{3}$, which is cubic. At one time, acicular particles of $\alpha \mathrm{Fe}$ were used to make 'Lodex', rather feeble elongated single domain magnets with energy products of up to $20 \mathrm{kJm}^{-3}$. [26]

What then is a permanent magnet? It is a piece of hard or semi-hard material with a useful energy product that retains its magnetization almost indefinitely. We might take isotropic bonded ferrite with $(B H)_{\max }=5 \mathrm{kJm}^{-3}$ and $M_{\mathrm{s}}=0.14 \mathrm{MAm}^{-1}$ as the lower limit for adequate magnetization. But we need to make a distinction between magnets made, for example, from hexagonal ferrite and alnico. Ferrite magnets can be any shape we want, and we can often optimize machine design by using magnets with $\mathcal{D} \approx 1 / 2$ without worrying much about them getting demagnetized. This is not true for alnico, which is below the shape barrier where the lack of internal anisotropy means that we have to accept constraints on the shape of the magnets that can be employed. Sometimes this is unimportant, but for others applications elaborate design rules have been developed to work around the inherent shortcomings of the material. Materials in the first category are hard, with $\kappa>1$, and the permanent magnets made from them might be qualified as 'true' or 'compact'. Otherwise, as with Pluto and the other planets, permanent magnets 
made from alnico, or another semihard material with $\kappa<1$ could be downgraded to 'semi-permanent' or 'conditional' status.

By drawing the line for magnetic hardness at $\kappa=1$, the rule of thumb for possible success in compact permanent magnet development is that the material should be hard. This is borne out by the examples in Table 1 . Alnicos, with $\kappa \approx 0.3$ are semihard, but they are rare-earth-free and have a relatively high magnetization. Several transition-metal alloys presently being investigated as potential rare-earth-free permanent magnets are also of this semi-hard type. Anisotropies of $1-2 \mathrm{MJm}^{-3}$, claimed as surprising or giant by comparison with soft magnetic materials, are inadequate to lift them into the hard category where they could compete on a level playing field with the materials at the top of the Table.

The question of how much anisotropy we need for a magnet of compact shape can be approached using Eq. (7) and the condition $H_{\mathrm{c}}>M_{\mathrm{s}} / 2$, which yields, for rectangular hysteresis loops, the optimum hardness

$$
\kappa>\frac{1}{2} \sqrt{\frac{1+2 \mathcal{D}_{\mathrm{eff}}}{\alpha}}
$$

This equation shows that the necessary hardness depends on the Kronmüller factor $\alpha$. Taking $\alpha=0.3$ as a realistic upper limit and estimating $\mathcal{D}_{\text {eff }}=1 / 3$ yields $\kappa_{0}=1.2$, in agreement with the above rule of thumb.

Energy-product development in the semihard zone is a very challenging task. For small anisotropies,

$$
(B H)_{\max }=c\left(\alpha K_{1}-1 / 2 \mu_{\mathrm{o}} \mathcal{D}_{\mathrm{eff}} M_{\mathrm{s}}^{2}\right)
$$

where $c$ depends on the loop shape ( $c=2$ for rectangular loops, $c=1 / 2$ for straight lines in the second quadrant). The equation shows that a sufficiently high $\alpha$ and a small or negative $\mathcal{D}_{\text {eff }}$ are necessary. In alnico magnets, which consist of elongated soft regions of FeCo embedded in a nonmagnetic NiAl matrix [2,27,28] the shape anisotropy of the elongated FeCo inclusions acts like a positive contribution $K_{1}$ s to the anisotropy in Eq. 
(10). For idealized alnico with a $2 / 3$ volume fraction of $\mathrm{FeCo},(B H)_{\max }=\mu_{\mathrm{o}} M_{\mathrm{s}}{ }^{2} / 12$ [27] and $\kappa=0.29$. Depending on details of the $\mathrm{FeCo} / \mathrm{NiAl}$ interface, there may be a positive or negative interfacial contribution to $K_{1}$ for alnicos, which are respectable conditionally permanent magnets, albeit with low coercivity.

\section{New Materials}

Next, we comment briefly on some rare-earth free materials listed in Table 2 that are under consideration for permanent magnet development. They are Mn, Fe or Cobased.

Manganese is interesting [20]. It is a cheap $3 d$ metal that exhibits a bigger moment than iron on certain sites in some compounds. In the cubic, ferromagnetic half-Heusler $\mathrm{NiMnSb}$, for example $\left(T_{\mathrm{C}}=455^{\circ} \mathrm{C}\right)$ the $\mathrm{Mn}$ moment is $3.8 \mu \mathrm{B}$. Unfortunately when manganese atoms are packed closer together, the moment is reduced and the exchange tends to be antiferromagnetic because the Mn $3 d$ band is almost half full. There are a few uniaxial ferro- or ferrimagnetic Mn alloys with modest polarizations of less than a tesla. Tetragonal carbon-stabilized MnAl magnets with energy products of $\approx 50 \mathrm{kJm}^{-3}$ were developed in the 1970s [2]. First-principles calculations confirmed strongly ferromagnetic exchange for the short in-plane Mn-Mn distances [29, 30] The material is potentially cheap and light, and could repay further research, especially if a magnetic substitution for $\mathrm{Al}$ was found that did not couple antiparallel to the $\mathrm{Mn}$.

Hexagonal $\mathrm{MnBi}$ has now been developed to the point where oriented magnets with energy products of $46 \mathrm{kJm}^{-3}$ [31] and $62 \mathrm{kJm}^{-3}$ [32] have been prepared from ball-milled powders. The material is unstable above $200^{\circ} \mathrm{C}$. Bismuth supply could be problematic if large quantities were required.

$\mathrm{Mn}_{2} \mathrm{Ga}$ is the end member of the tetragonal $\mathrm{Mn}_{3-\mathrm{x}} \mathrm{Ga}$ solid solution where many substitutions are possible. The relatively low moment is attributed to a ferrimagnetic structure. Good coercivity is associated with modest magnetization $[33,34]$, but oriented magnets have not yet been prepared. The cost of Ga will be prohibitive unless a substitute is found. 
Tetragonal FeNi (tetrataenite), originally discovered in Ni-Fe meteorites, formed with cooling times in excess of a billion years, is now being explored as a potential manmade permanent magnet [35-37]. L10 FeNi has a high magnetization, but insufficient anisotropy $\left(1.3 \mathrm{kJm}^{-3}\right)$ to raise it out of the semi-hard category. The obstacles are inadequate anisotropy, modest coercivity $(120 \mathrm{mT})$ and difficulty in achieving and retaining the $L 1_{0}$ atomic order. Studies have focused on natural samples and thin films, although Néel and coworkers showed in 1964 that it was possible to induce L10 order in a single-crystal by neutron irradiation in an applied field [38].

The tetragonal interstitial nitride $\alpha " \mathrm{Fe}_{16} \mathrm{~N}_{2}$ [39] became well-known, after claims of an exceptionally large magnetization in thin films. Recently pure powder has been produced and characterized [40]. The magnetization is similar to that of $\alpha \mathrm{Fe}$, and the material is unstable and semi-hard $\kappa \approx 0.5$. Dense bulk magnets have not been produced. Orthorhombic $\mathrm{Fe}_{3} \mathrm{C}$ is stable, but it also lacks the anisotropy that would be needed to make it a serious contender.

Cobalt metal has a magnetization that is exceptionally stable. Some coercivity has been developed by depositing short wires in a microporous alumina template [41,42], but the combination of shape and magnetocrystalline anisotropy is insufficient to make a hard magnet, even though the magnetization is reduced by $\sim 60 \%$ due to the inert volume of the membrane. Template-free nanorods exhibit a magnetization similar to that of bulk cobalt, and coercivity of up to $0.89 \mathrm{~T}$ [43]. $\mathrm{Co}_{2} \mathrm{C}$ is orthorhombic, and has been produced in the form of coercive nanoparticles, with a relatively small magnetization [44]. The magnetization of $\mathrm{CoFe}_{2} \mathrm{C}$ nanoparticles is slightly higher, but they exhibits even less coercivity, despite a questionable claim of exceptional magnetocrystalline anisotropy in this compound [45].

YCos has very large anisotropy, so it is a good candidate for permanent magnet development. Ball-milled powders with a small iron substitution have a powder energy product of $140 \mathrm{kJm}^{-3}$, and isotropic magnets with $(B H)_{\max }=64 \mathrm{kJm}^{-3}$ have been prepared from them [46]. This could be substantially improved in oriented magnets. The value of this material depends on the availability of yttrium, and its cost relative to samarium. Other candidates for non-rare-earth permanent magnets include $\mathrm{Zr}_{2} \mathrm{Co}_{11}$ [47] and orthorhombic $\mathrm{HfCo}_{7}$ [48], which have magnetizations comparable to $\mathrm{SmCo}$. 
The performance of rare-earth transition-metal intermetallics is difficult to beat, and it is unlikely that any of these new materials will completely replace $\mathrm{Nd}_{2} \mathrm{Fe}_{14} \mathrm{~B}$ and $\mathrm{Sm}$ Co magnets. However, $\mathrm{Nd}_{2} \mathrm{Fe}_{14} \mathrm{~B}$ is presently used for many applications where moderate energy products are sufficient, opening the field for a gap magnet. The key is sufficient anisotropy and magnetization at reasonable cost.

\section{Other approaches}

An approach that has been investigated for many years has been to make two-phase nanocomposites of a high-moment soft phase exchange coupled to a high-anisotropy hard phase [49]. These exchange-spring magnets [50] can, in principle, average the magnetization and anisotropy of the two constituents [13]. They have not yet realized their promise, due to the difficulty in creating a bulk sample where the two constituents are intergrown on the requisite scale of less than $10 \mathrm{~nm}$. Advances in nanostructured permanent magnet research have been reviewed by Poudyal and Liu [51], and there is a comprehensive review of nanoscale magnetism [52].

A working point in the second quadrant of the hysteresis loop does not necessarily demand large hysteresis. It is possible to shift the loop of a ferromagnet to negative fields by exchange bias, a phenomenon familiar in thin film heterostructures used in spin electronics [4]. It has recently been shown that it is possible to achieve exchange bias in bulk $\mathrm{Mn}_{2-\mathrm{x}} \mathrm{Pt}_{\mathrm{x}} \mathrm{Ga}$ where coherent antiferromagnetic inclusions precipitate out in a ferromagnetic matrix [53]. The method is interesting, but the challenge is to find a materials system that shows an adequate Curie temperature, useful magnetization and an appropriate phase diagram with a strongly anisotropic antiferromagnetic phase. Even so, there is no benefit compared to an exchange-coupled hard-soft nanocomposite [29], other than perhaps reduced materials costs, because here we are replacing the hard ferromagnetic component by a hard antiferromagnetic component, which makes no contribution to the net magnetization.

A quite different approach to permanently trapping magnetic flux is to use a granular high-temperature superconductor [54]. The record flux trapped in this way is 17.2 $\mathrm{T}$ [55], an order of magnitude greater than for the best conventional permanent magnets. The energy product is enormous, of order $50 \mathrm{MJm}^{-3}$, neglecting the steel sheaths 
and ancilliary cooling equipment. There may be potential applications in high-power motors, generators and magnetically-levitated flywheels if the problems of charging and cooling the magnets can be resolved.

\section{Conclusions}

The amount of anisotropy required to make a permanent magnet scales with the square of the magnetization. Past experience and the present analysis indicate that magnetocrystalline anisotropy significantly greater than $\mu_{0} M_{\mathrm{s}}{ }^{2}(\kappa>1)$ is needed to make magnets of any desired shape from a hard magnetic material, particularly those with $\mathcal{D}=$ $1 / 2$ which are most efficient at storing magnetostatic energy. Specific values recommended for materials with $\mu_{0} M_{\mathrm{s}}=1.0,1.5$ and $2.0 \mathrm{~T}$ are at least $1.2,2.6$ and $4.6 \mathrm{MJm}^{3}$, respectively. The latter is close to the limit of what can be achieved without rare earths. Table 2 shows that the necessary values are achieved in some candidate materials, but not in others such as $L 1_{0} \mathrm{NiFe}, \alpha " \mathrm{Fe}_{16} \mathrm{~N}_{2}$ or $\mathrm{Fe}_{3} \mathrm{C}$.

When such anisotropy is not available, the material is semihard, and the best that can be expected is that it might be possible to make permanent magnets in a restricted range of shapes with sufficiently small demagnetizing factors. These magnets cannot come anywhere near the theoretical energy product of $1 / 4 \mu_{0} M_{\mathrm{s}}^{2}$, and they make it awkward to engineer electrical machines. There would have to be a compelling cost advantage to justify a development effort for a new semihard material to compete with alnico. Without a clear strategy concerning microstructure, there is little hope of developing such materials into competitive permanent magnets

\section{Acknowledgements}


RS acknowledges support from ARPA-E (Argonne/Delaware), DREaM, and DOE BES. (DE-FG02-04ER46152). and discussions with B. Balamurugan, S. Constantinides, M. J. Kramer, L. H. Lewis, P. Manchanda, R. W. McCallum, S. G. Sankar, D. J. Sellmyer, and J. E. Shield. JMDC acknowledges support from SFI Contract 13/ERC/12561 and the EU FP7 ROMEO Project, and comments from M. Venkatesan and P. Tozman. 


\section{Figure Captions}

Fig. 1. Plots of anisotropy $K_{1}$ as a function of polarization of $\mu_{0} M_{\mathrm{s}}$ for a wide variety of magnetic materials with uniaxial anisotropy. The anisotropy for the alnicos (red points) is shape anisotropy (Eq 3). The solid and dotted lines correspond to $\kappa=1,0.1$ and $\kappa=$ $1 / 2$ respectively. Hard materials in the bright green can be used to make efficient magnets of any shape. Semihard materials in the pale green area can be used to make oriented magnets with a severely shape-limited energy product.

Fig. 2. Permanent magnets of different shapes. (a) thin film with in-plane magnetization $\mathcal{D} \approx$ 0.01 (b) thin film with perpendicular magnetization $\mathcal{D} \approx 0.99$, (c) horseshoe-like toroid with $\mathcal{D} \approx 0.05$, and (d) optimized compact magnet $\mathcal{D} \approx 0.50$

Fig. 3. Analysis of ideal rectangular hysteresis loops of magnets with $\mathcal{D} \approx 0,1 / 2$ and 1 to deduce the energy products of $0,1 / 4 \mu_{0} M_{\mathrm{s}}^{2}$ and 0 , respectively. The dotted lines show the effect of correcting the applied field for the demagnetizing effect. 
Table 1. Room-temperature intrinsic properties of some hard and semi-hard materials used for oriented permanent magnets

\begin{tabular}{|l|r|r|r|r|}
\hline Material & \multicolumn{1}{|c|}{$T_{\mathrm{C}}(\mathrm{K})$} & \multicolumn{1}{|c|}{$\mu_{\mathrm{o}} M_{\mathrm{s}}(\mathrm{T})$} & $\begin{array}{c}K_{1} \\
\left(\mathrm{MJ} / \mathrm{m}^{3}\right)\end{array}$ & $\kappa$ \\
\hline $\mathrm{Nd}_{2} \mathrm{Fe}_{14} \mathrm{~B}$ & 588 & 1.61 & 4.9 & 1.54 \\
\hline $\mathrm{Sm}_{2} \mathrm{Fe}_{17} \mathrm{~N}_{3}$ & 694 & 1.54 & 8.9 & 2.17 \\
\hline $\mathrm{Sm}_{2} \mathrm{Co}_{17}$ & 1190 & 1.21 & 4.2 & 1.89 \\
\hline $\mathrm{SmCo}_{5}$ & 1020 & 1.05 & 17.0 & 4.40 \\
\hline $\mathrm{CoPt}$ & 840 & 1.01 & 4.9 & 2.47 \\
\hline $\mathrm{BaFe}{ }_{12} \mathrm{O}_{19}$ & 740 & 0.48 & 0.33 & 1.35 \\
\hline Alnico 5 & 1240 & $(1.35) 1.40$ & 0.32 & 0.45 \\
\hline Alnico 8 & 1220 & $(0.82) 1.20$ & 0.24 & 0.46 \\
\hline Alnico 9 & 1260 & $(1.06) 1.25$ & 0.26 & 0.45 \\
\hline
\end{tabular}

Remanences $\boldsymbol{\mu}_{\mathbf{0}} M_{\mathbf{r}}$ for alnico magnets are in parentheses.

Table 2. Room-temperature intrinsic properties of candidate rare-earth free magnet materials

\begin{tabular}{|l|l|l|l|l|l|l|l|l|l|}
\hline & $\mathrm{MnAl}$ & $\mathrm{MnBi}$ & $\mathrm{Mn}_{2} \mathrm{Ga}$ & $\mathrm{FeNi}$ & $\mathrm{Fe}_{16} \mathrm{~N}_{2}$ & $\mathrm{Fe}_{3} \mathrm{C}$ & $\mathrm{Co}$ & $\mathrm{Co}_{2} \mathrm{C}$ & $\mathrm{YCO}_{5}$ \\
\hline Structure & $L 1_{0}$ & $B 8_{1}$ & $D 0_{22}$ & $L 1_{0}$ & $\alpha^{\prime \prime}$ & $D 0_{11}$ & $A 3$ & $C 23$ & $D 2_{d}$ \\
\hline$T_{\mathrm{C}}(\mathrm{K})$ & 650 & 633 & $>770$ & $>820$ & 810 & 560 & 1360 & 510 & 987 \\
\hline$\mu_{0} M_{s}(\mathrm{~T})$ & 0.75 & 0.73 & 0.59 & 1.59 & 2.10 & 1.36 & 1.81 & 0.7 & 1.06 \\
\hline$K_{1}\left(\mathrm{MJm}^{-3}\right)$ & 1.7 & 0.90 & 2.35 & 1.3 & 1.0 & 0.45 & 0.41 & 1.1 & 6.5 \\
\hline$\kappa$ & 1.95 & 1.46 & 2.35 & 0.80 & 0.53 & 0.56 & 0.45 & 1.7 & 2.7 \\
\hline
\end{tabular}




\section{References}

[1] S. Chikazumi, Physics of Magnetism, Wiley, New York 1964.

[2] R. A. McCurrie Ferromagnetic Materials; Structure and Properties Academic Press, London 1994

[2] R. Skomski and J. M. D. Coey, Permanent Magnetism, Bristol: Institute of Physics, 1999.

[4] J. M. D. Coey Magnetism and Magnetic Materials, Cambridge University Press 2010

[5] W. Jellinghaus, Z. tech. Physik 17 (1936) 33.

[6] T. Klemmer, D. Hoydick, H. Okumura, B. Zhang, and W. A. Soffa, Scripta Met. Mater. 33 (1995) 1793.

[5] C. Kooy and U. Enz, Philips Res. Repts. 15 (1960) 7.

[8] M. Sagawa, S. Fujimura, H. Yamamoto, and Y. Matsuura, IEEE Trans. Magn. 20 (1984) 1584.

[9] M. Sagawa, S. Hirosawa, H. Yamamoto, S. Fujimura, and Y. Matsuura, Jpn. J. Appl. Phys. 26 (1987) 785.

[10] J. F. Herbst, Rev. Mod. Phys. 63 (1991) 819.

[9] K. Kumar, J. Appl. Phys. 63 (1988) R13.

[10] J. M. D. Coey and H. Sun, J. Magn. Magn. Mater. 87 (1990) L251.

[13] J. M. D. Coey, IEEE Trans. Magn. 49 (2011) 4671.

[14] J. M. D. Coey, Scripta Materialia 67 (2012) 524

[15] N. Jones, Nature 472 (2011) 22.

[16] O. Gutfleisch, M. A. Willard, E. Brück, C. H. Chen, S. G. Sankar, and J. P. Liu, Adv. Mater. 23 (2011) 821.

[14] R. W. McCallum, L. H. Lewis, R. Skomski, M. J. Kramer, and I. E. Anderson, Ann. Rev. Mater. Res. 44 (2014) 451.

[18] X. Zhao, M. C. Nguyen, W. Y. Zhang, C. Z. Wang, M. J. Kramer, D. J. Sellmyer, X. Z. Li, F. Zhang, L. Q. Ke, V. P. Antropov, and K. M. Ho, Phys. Rev. Lett. 112 (2014) 045502. 
[19] P. Kumar, A. Kashyap, B. Balamurugan, J. E. Shield, D. J. Sellmyer, and R. Skomski, J. Phys.: Condens. Matter 26 (2014) 064209.

[20] J. M. D. Coey, J. Phys. Cond Matter 26 (2014) 064211

[21] Consider $\left.\nabla_{\mathbf{A}} \times \mathbf{H}\right)=\mathbf{B} \cdot \mathbf{H}$, which follows from $\mathbf{B}=\nabla_{\mathbf{A}}$ and $\mathbf{H}=-\nabla_{\mathrm{m}}$. Since $\left.\int \nabla_{\mathbf{A}} \times \mathbf{H}\right) \mathrm{d} V$ $=\int(\mathbf{A} \times \mathbf{H}) \mathrm{d} \mathbf{S}$ and the respective $\mathbf{A}$ and $\mathbf{H}$ fields decay as $1 / r^{2}$ and $1 / r^{3}$ in infinity, the surface integral and therefore $\int \mathbf{B} \cdot \mathbf{H} \mathrm{d} V$ are equal to zero.

[22] Note that the total magnetostatic self energy, or dipole-dipole energy, $-1 / 2 \mu_{\mathrm{o}} \int \mathbf{M} \cdot \mathbf{H} \mathrm{dV}=1 / 2 \mu_{\mathrm{o}}$ $\mathcal{D} M^{2} \mathrm{~V}$, is the sum of $E_{\mathrm{a}}=1 / 2 \mu_{\mathrm{o}} \mathcal{D}(1-\mathcal{D}) M^{2} V$ and $E_{\mathrm{i}}=1 / 2 \mu_{\mathrm{o}} \mathcal{D}^{2} M^{2} V$.

[23] H. Sepheri-Amin, T. Ohkubo, M. Gruber, T. Shrefl and K. Hono, Scripta Materialia 89 (2014) 29

[24] H. Kronmuller, physica status solidi (b) 144 (1987) 385

[25] R. Skomski, A. Kashyap, A. Solanki, A. Enders, and D. J. Sellmyer, J. Appl. Phys. 107 (2010) 09A735.

[26] R. J Parker, Advances in Permanent Magnetism, Wiley-Interscience, New York 1990 p320

[27] R. Skomski, Y. Liu, J. E. Shield, G. C. Hadjipanayis, and D. J. Sellmyer, J. Appl. Phys. 107 (2010) 09A739.

[19] L. Zhou, M. K. Miller, P. Lu, L. Ke, R. Skomski, H. Dillon, Q. Xing, A Palasyuk, M. R. McCartney, D. J. Smith, S. Constantinides, R. W. McCallum, I. E. Anderson, V. Antropov, and M. J. Kramer, Acta Materialia 74 (2014) 224.

[29] R. Skomski, P. Manchanda, P. Kumar, B. Balamurugan, A. Kashyap and D. J. Sellmyer, IEEE Trans Magn. 49 (2013) 3215

[30] P. Manchanda, P. Kumar, A. Kashyap, M. J. Lucis, J. E. Shield, A. Mubarok, J. Goldstein, S. Constantinides, K. Barmak, L.-H. Lewis, D. J. Sellmyer, and R. Skomski, IEEE Trans Magn. 49 (2013) 5194.

[31] N. V. Rama Rao, A. M. Gabay and G. C. Hadjipanayis, J. Phys D 46 (2013) 062001

[32] J. Cui, J. P. Choi, G. Li , E. Polikarpov et al, J. Phys: Condens Matter 26 (2014) 064212

[33] H. Kurt, K. Rode, M. Venkatesan, P. Stamenov and J. M. D. Coey, Phys. Status Solidi B 248 (2011) 2338 
[34] A A El-Gendy and G. Hadjipanayis, J. Phys. Chem. C 119 (2015) 8898

[35] L. H. Lewis, A. Mubarok, E. Poirier, N. Bordeaux, P. Manchanda, A. Kashyap, R. Skomski, J. Goldstein, F. E. Pinkerton, R. K. Mishra, R. C. Kubic Jr., and K. Barmak, J. Phys.: Condens. Matter 26 (2014) 064213.

[20] L. H. Lewis, F. E. Pinkerton, N. Bordeaux, A. Mubarok, E. Poirier, J. I. Goldstein, R. Skomski, and K. Barmak, IEEE Magnetics Lett. 5 (2014) 5500104.

[37] T. Kojima, M. Ogiwara, M. Mizuguchi, M. Kotsugi et al, J. Phys; Condens Matter 26 064207 (2014)

[38] L. Néel, J. Paulevé, R. Pauthenet, J. Laugier, and D. Dautreppe: J. Appl. Phys, 35. (1964) 873

[39] J. M. D. Coey, K. O'Donnell, Q. Qinian, E. Touchais, and K. H. Jack, J. Phys.: Condens. Matter 6 (1994) L23.

[40] T. Ogawa, Y. Ogata, R. Gallage, N. Kobayashi, N. Hayashi et al, Appl. Phys. Express 6 073007 (2013)

[41] I. Dobosz, W. Gumowska and M. Kzapkiewicz, J. Solid State Electrochem.18 2963 (2014)

[42] R Lanvin, J. C. Denardin, A. Cortez, H. Gomez, M. Cornejo and G Gonzalez, Mol. Cryst. Liq. Cryst 521, 293 (2010)

[43] F. Dumestre, B. Chaudret, C Amiens, M-C Fromen, M-J Casanove, P. Renaud and P. Zurcher, Angew Chem Int Ed 41 (2002) 4286

[44] V. G. Harris, Y. Chen, A. Yang, S. Yoon, Z. Chen et al J. Phys D: Appl. Phys 43 (2010) 165003

[45] A. A. El-Gendy, M. Berlino, D. Clifford, M Qian, S. N. Khanna and E. E. Carpenter Appl Phys Letters 106 (2015) 213109

[46] P. Tozman, P. Tozman, M. Venkatesan, G. Zickler, J. Fidler and J. M. D. Coey, Appl. Phys. Lett. 107 (2015) 032405

[47] W Y Zhang, X. Z. Li, S. Volloppolly, R. Skomski, J. E. Shield and D. J. Sellmyer et al 2013 J. Phys. D: Appl. Phys. 46 (2013) 135004

[48] B. Balamurugan, B. Das, V. R. Shah, R. Skomski, X. Z. Li, and D. J. Sellmyer, Appl. Phys. Lett. 101 (2012) 122407. 
[49] R. Skomski and J. M. D. Coey, Phys. Rev. B 48 (1993) 15812.

[50] E. F. Kneller and R. Hawig, IEEE Trans. Magn. 27 (1991) 3588.

[51] N. Poudyal and J. P. Liu, J. Phys. D: Appl. Phys 46 (2013) 043001

[52] R. Skomski, J. Phys.: Condens. Matter 15 (2003) R841.

[53] A. J. Nayak, M. Nicklas, S. Chadov, P. Khuntia, C. Sheckhar et al, Nature Materials, 14 (2105) 679

[54] L. Schultz, G. Krabbes, G. Fuchs, W. Pfeiffer and K-H Müller, Z. f. Metallkunde, 93 (2002) 1057

[55] J. H. Durrell, A. R. Dennis, J. Jaroszynski, M. D. Ainslie, K. G. B. Palmer et al Supersond Sci Technol 27 (2014) 082001 
$M_{\mathrm{S}}\left(\mathrm{MA} \mathrm{m}^{-1}\right)$

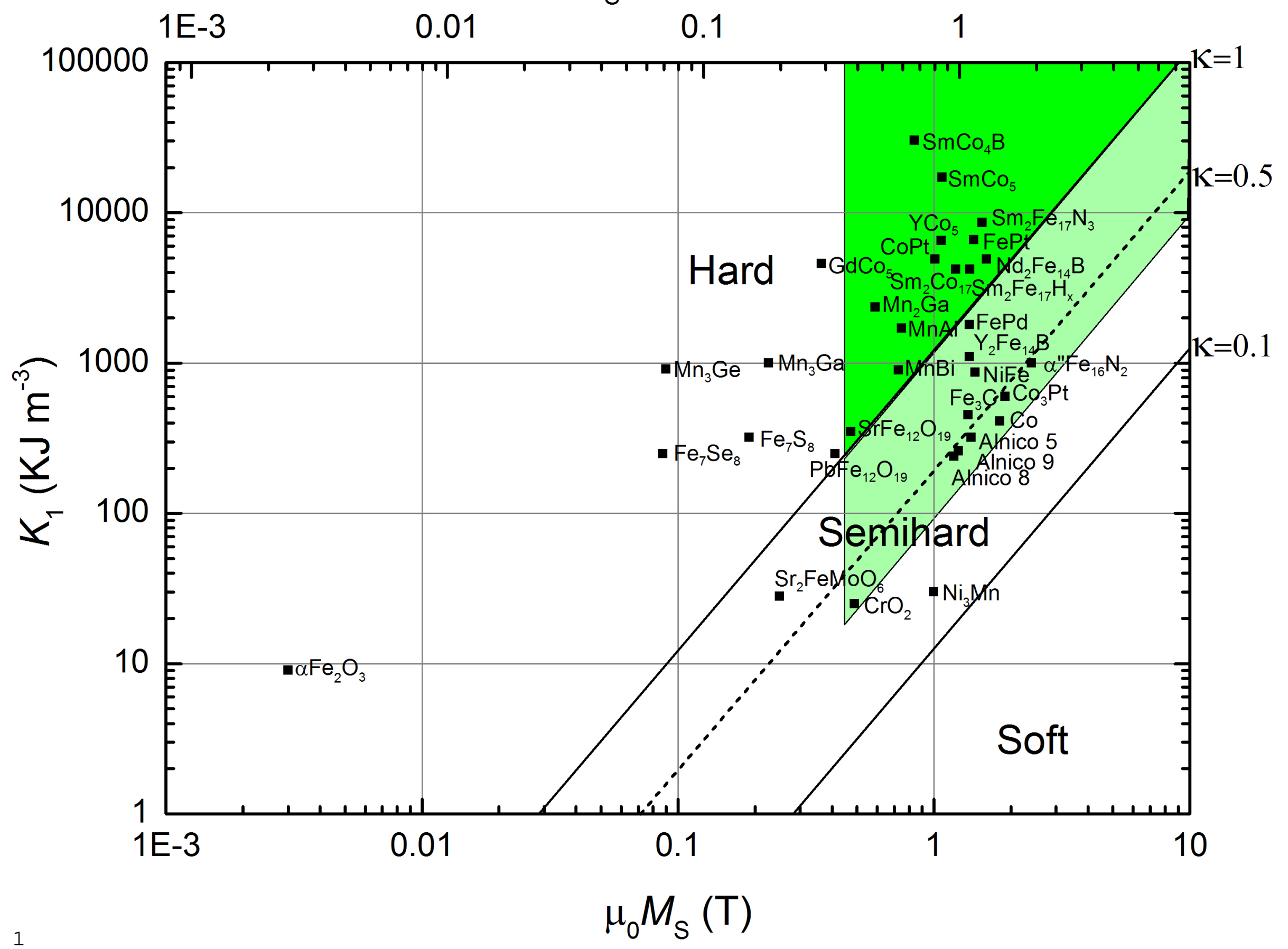



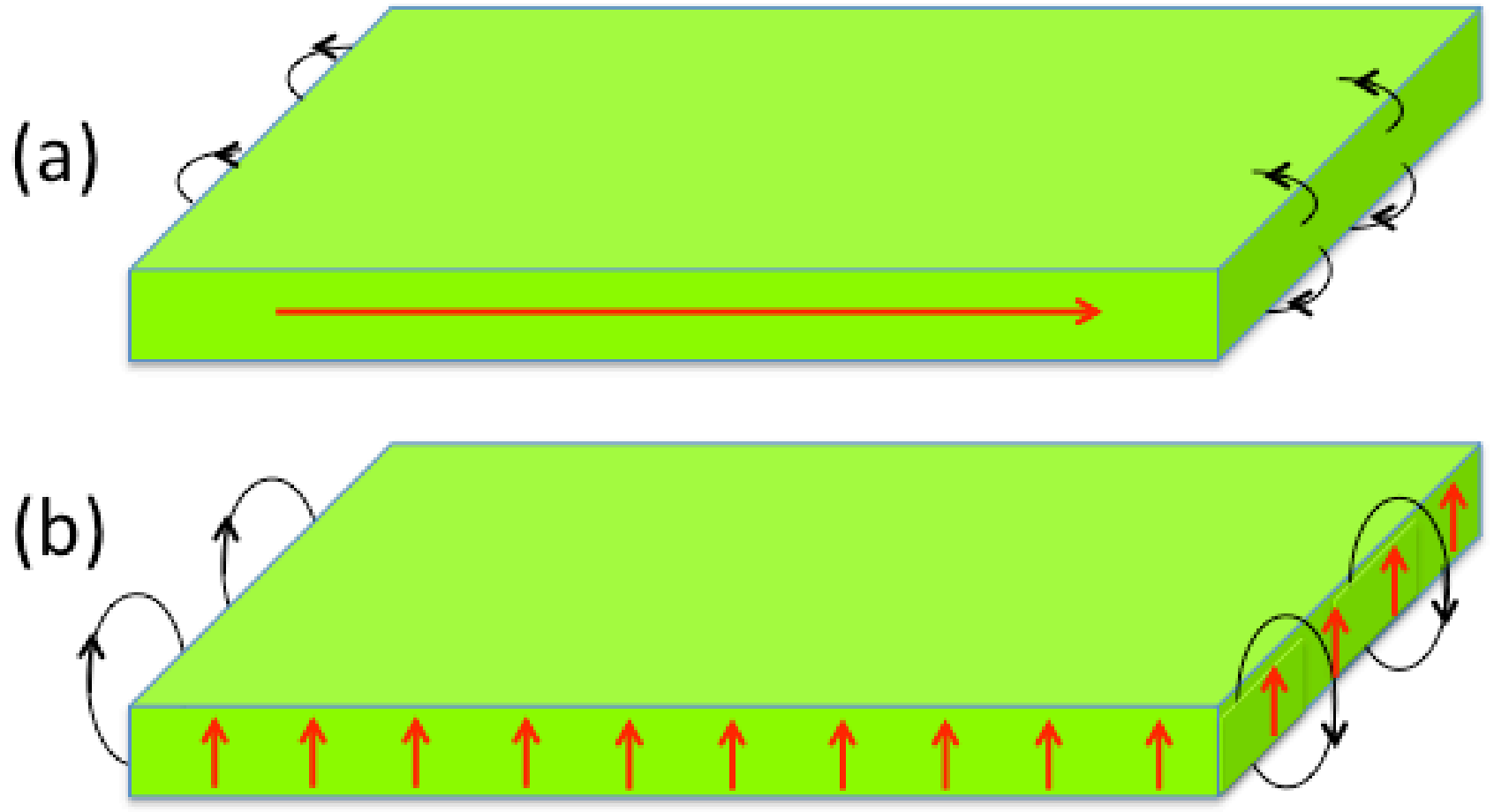

(c)

figure 2

(d)

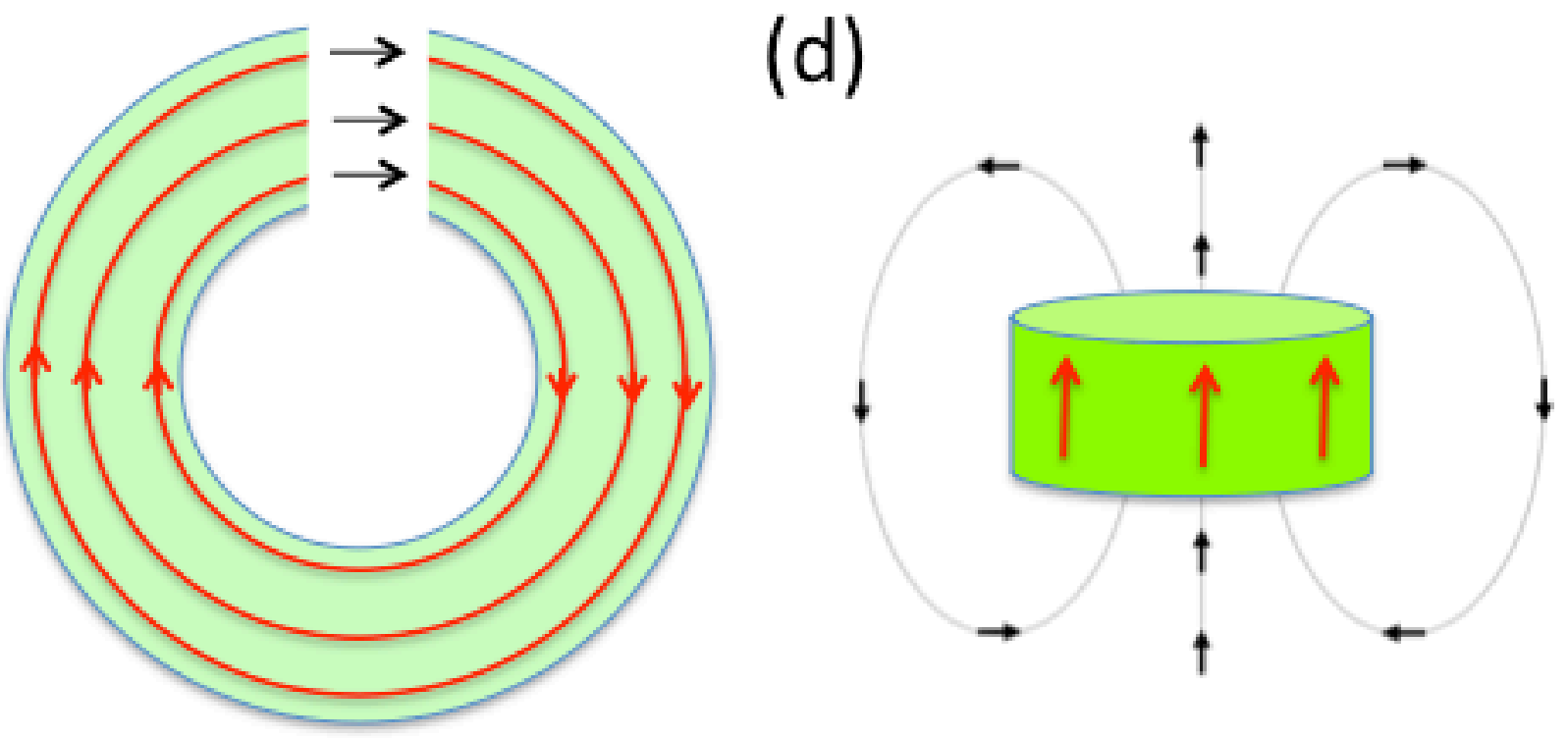




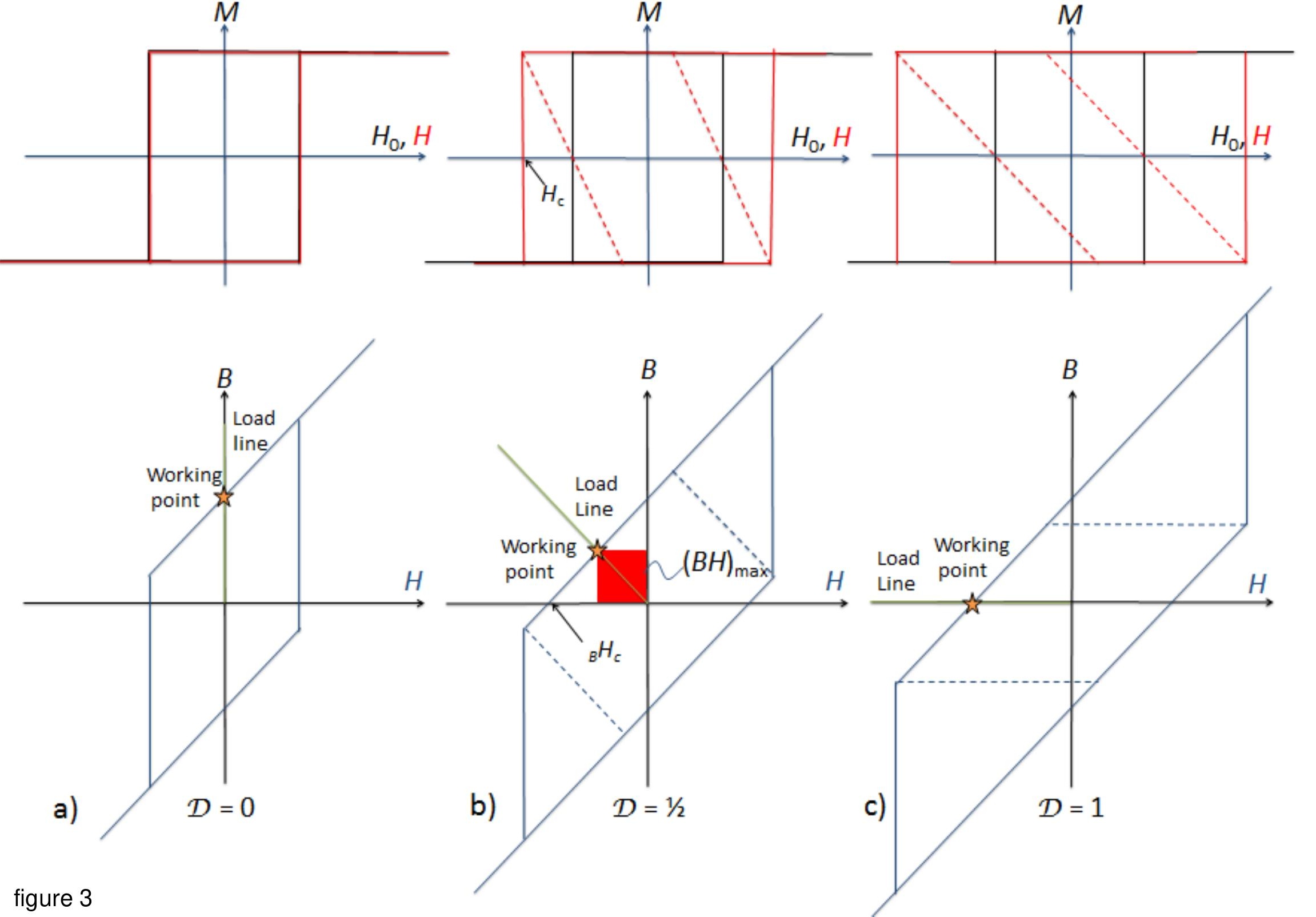

